\title{
A Scheme of Dynamic Bandwidth Allocation for Switching FC-AE-1553 Network
}

\author{
Shaojun $\mathrm{WU}^{1,3^{*}}$, Liqian WANG ${ }^{2}$,Yueying $\mathrm{ZHAN}^{1}$, Guangheng $\mathrm{ZHAO}^{1}$, Jin \\ WANG $^{2}$ \\ ${ }^{1}$ Technology and Engineering Center for Space Utilization, Chinese Academy of Science, Beijing \\ 100094, China; \\ ${ }^{2}$ State Key Laboratory of Information Photonics and Optical Communications, Beijing University of \\ Posts and Telecommunications. Beijing 100876, China; \\ ${ }^{3}$ University of Chinese Academy of Sciences, Beijing 100049, China \\ *wushaojun@csu.ac.cn
}

Keywords: Switching FC-AE-1553, periodical and concurrent DBA, periodic data, application data, burst data

\begin{abstract}
In order to meet the requirement of space payload system and the data transmission, a scheme of periodical and concurrent dynamic bandwidth allocation (DBA) of switching FC-AE-1553 network was proposed based on both theoretical analysis and simulation approaches. Compared with traditional solutions, the network throughput was increased by 10 times, and the time delay was decreased by one order of magnitude.
\end{abstract}

\section{Introduction}

With the continuous expansion of the number of space stations, the species of payload, and the transmission rate, the control scheme become even more complicated, which propose high demanding request to the data processing capability and system reliability[1-3].

FC-AE-1553 as one of the protocols FC-AE is considered as the candidate protocol of aerospace due to the high real-time, high-bandwidth, high reliability and compatible with MIL-STD-1553[4-7]. Switching FC-AE-1553 network as one of topologies of FC-AE-1553can realize the concurrent operation based on switch compared to the traditional FC-AE-1553 network. The bandwidth allocation of traditional FC-AE-1553 network is the non-concurrent sequential method and the method use the time division multiplexing to share the network bandwidth[8-9]. This method can't use the concurrent advantage of switching network, the system throughput is not improved and the bandwidth utilization is reduced. DING Fan, XIONG Hua-gang et al.[5] introduced a new method based on the traditional large-small periodical which can improve the bandwidth utilization. However, the method also use the time division multiplexing and is not based on the advantage of simultaneous operation multiple nodes.

Therefore, in order to meet the requirement of space payload system and the data transmission, the data type of payload was divided into periodic data, application data and burst data. In this paper, we present a periodical and concurrent dynamic bandwidth allocation (DBA) of switching FC-AE-1553 network through both theoretical analysis and simulation approaches. The performances of the proposed scheme is evaluated and discussed. Compared with traditional solutions, the network throughput increased by 10 times, and the delay decreased one order of magnitude. 


\section{Network architecture and payload data}

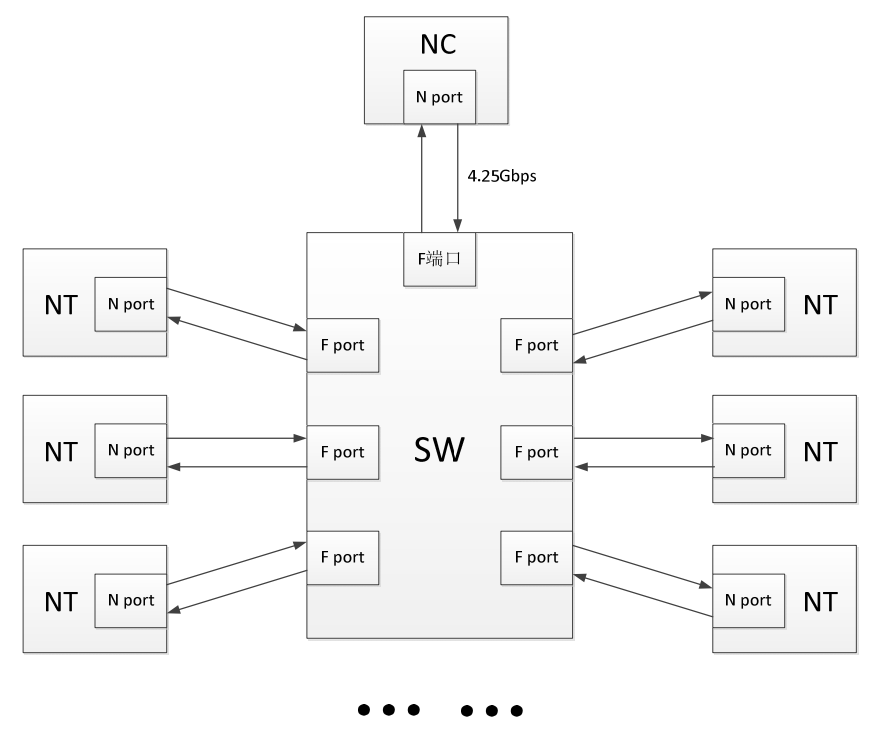

Fig. 1. Switching FC-AE-1553 network architecture

Figure 1 shows the architecture of switching FC-AE-1553 network which consists network controller (NC), network terminal (NT), switch and many fiber links. FC-AE-1553 network is the command/response network, and $\mathrm{NC}$ is the master node of the network. The data transmission was controlled by NC, and each data transmission is launched by NC. NT is the slave node and receives or transmits the data based on the NC command. The function of switch is forward the data. In our paper, the length of fiber among NC, NT and switch is $[0,300 \mathrm{~m}]$.

With the rapid development of space application system, the demanding of the exchanging information and sharing between electronic devices become higher. In order to meet the requirement of space payload system and the data transmission, the data types of payload were divided into periodic data, application data and burst data. Periodic data is accordance with a certain frequency transmitted data between NC and NT, such as timecode, telemetry data and power supply parameters. Application data is a large amount of data in some storage equipment, such as the data of science experiment or remote sensing. Bust data is the bust directive data which generated by NC. In the space payload system, the periodic data exists in NC and NT and not exists in NT and NT, application data exists in large-capacity equipment, and the burst data is only generated by NC and arrives at NT node.

\section{Principle and Proposed DBA Method}

In bus network, all data transmission between the nodes compete for the same link resource, and bandwidth utilization become lower and lower. However, in the switching network, competition and concurrent operation exist in the same link request. Therefore, we design a combination of static and dynamic periodical and concurrent dynamic scheduling scheme based on the features of the payload data. The bandwidth of FC-AE-1553 network is consider as the multiple timelines which are NC transmitting timeline, $\mathrm{NC}$ receiving timeline, NT transmitting timeline and $\mathrm{NT}$ receiving timeline. Periodic data use the static allocation, application data use the dynamic allocation and the burst data use the preemptive allocation scheme.

The application data which is sent by NC use the periodic and centralized request. The scheduling mechanism is the on-demand assignment algorithm based on first in first out. The application data which is sent by NT use the allocation algorithm based on credit value ordering, the command frame is generated by $\mathrm{NC}$ which include all the application data business requests, periodically broadcast to all the NT nodes, and statistical itself requirements. And the process of application allocation is following. Firstly, the transmission bandwidth timeline of NC is calculated and allocated. Secondly, the starting time of the NC-NT application data is fixed in the receiving timeline of NT. And then, the 
bandwidth of receiving timeline of all nodes can be concurrent and independent calculated and allocated. The order of scheduling is shown in Fig.2.

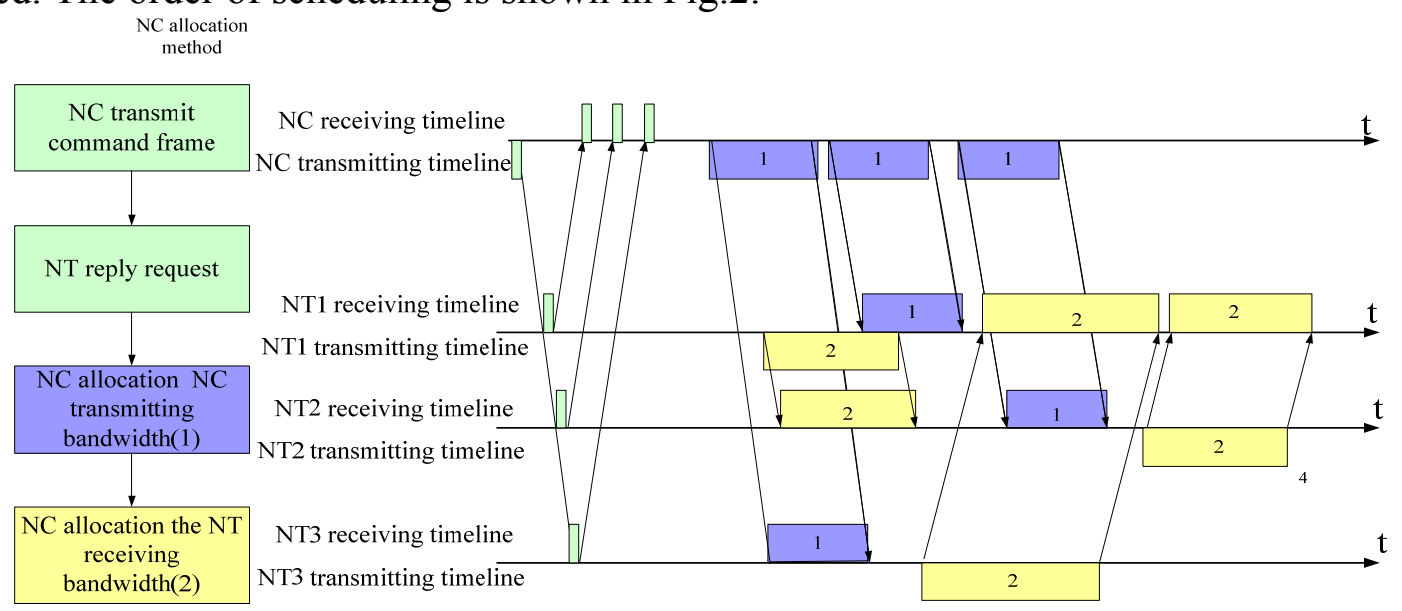

Fig. 2. The scheduling mechanism of application

The NC transmitting timeline use the assignment algorithm by priority, the largest delay of application data is firstly allocated and the larger delay is secondly allocated in the NC buffer. The receiving timeline of NC and NT use the assignment algorithm based on credit value queuing. The credit value is defined the difference between the actual bandwidth and the pre-set average bandwidth.

Due to the network traffic of periodic data is fixed and the periodical is strictly, the periodic data use the static allocation method. Therefore, the transmission of periodic data is not concurrent with any other data, which is shown in Fig.3.

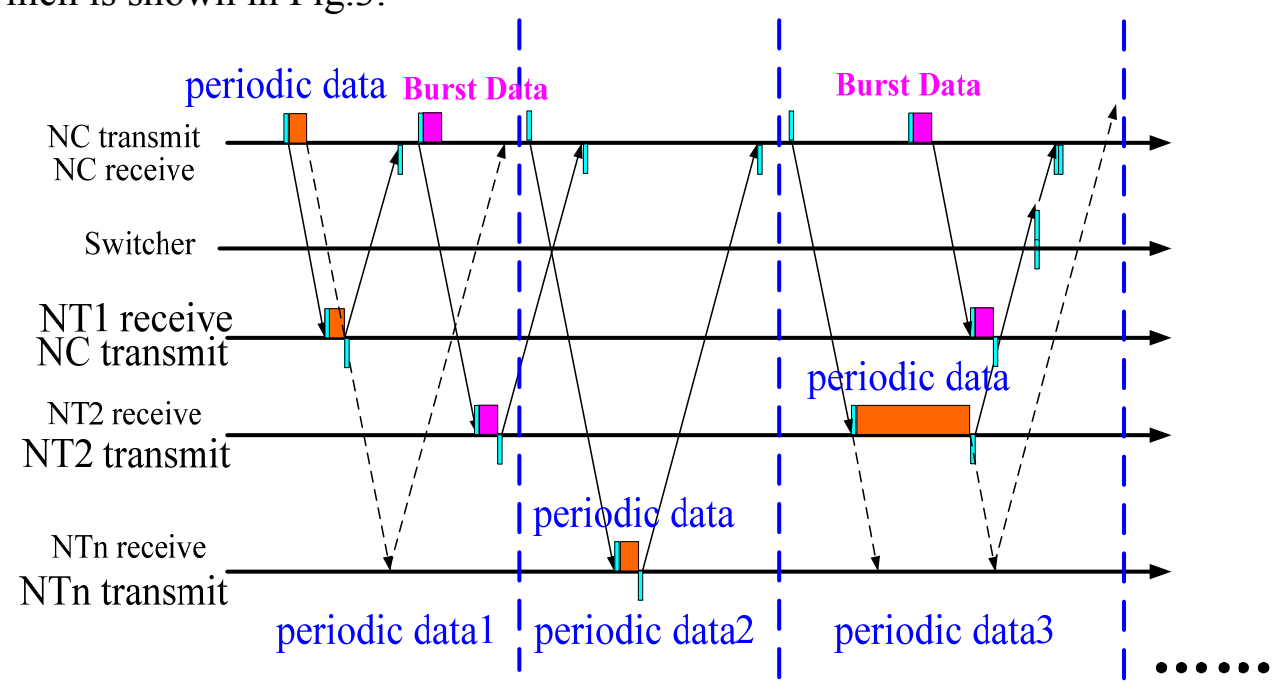

Fig. 3. The scheduling mechanism of periodic data and burst data

The burst data use the preemptive allocation because of the higher delay requirement, lower network traffic, and only NC-NT direction. The burst data insert the current FC frame and without waiting for the end of the exchange, the burst data can not interrupt the sending frame at same time. So the reservation slot should be added between the tasks of NT receiving timeline, which is shown in Fig.3.

\section{Simulations and discussions}

OPNET simulation framework software is employed to investigate the proposed scheme. In our switching FC-AE-1553 network simulation consists 1 NC nodes, 32 NT nodes and 1 switch, which is shown in Fig.4. 


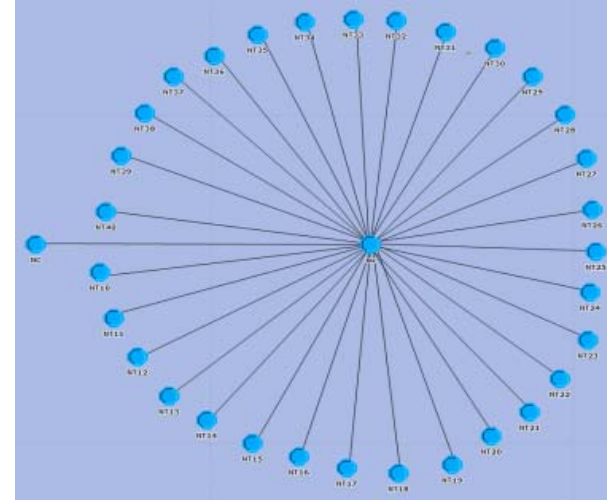

Fig. 4. Simulation system of switching FC-AE-1553 network

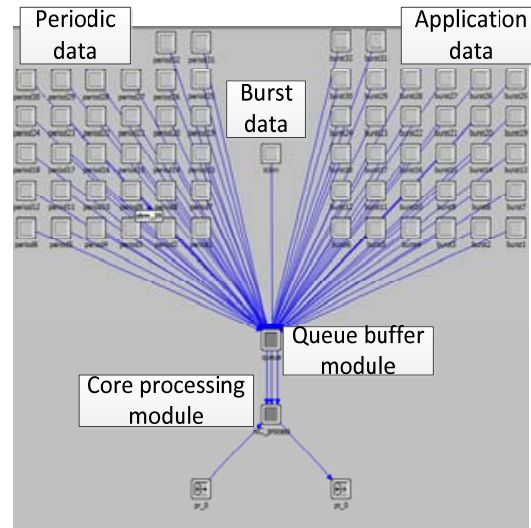

Fig. 5. The model of NC node

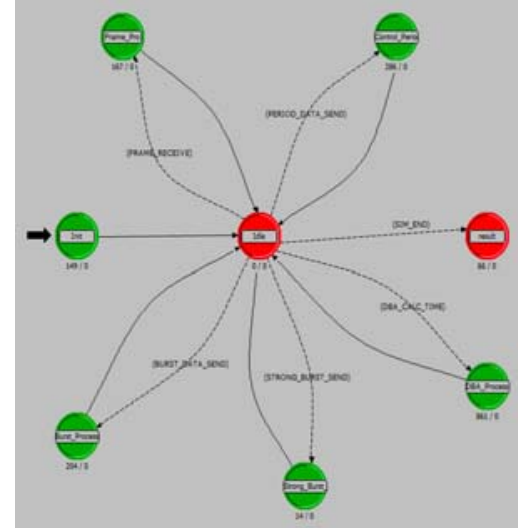

Fig. 6. The state machine of NC core process

In our paper, the data source exists in NC and NT nodes, the periodic data source can generate 512 byte FC frame, burst data source can generate FC frame which the length is 512 byte and the time interval obey the exponentially distributed, application use the ON/OFF model to realize the performance of self-similarity. The model of $\mathrm{NC}$ nodes is a four-layer structure, which is shown in Fig.5. FC-0 and FC-1 are built by point to point duplex link model and transceiver.FC-2 is realized by finite state machine which is shown in Fig.6. The NT node model is similar to NC which consists four-layer structure. Switch forward the FC frame and the largest number of nodes is set 32 .

Based on the features of the space payload system, we have discussed the network throughput, the largest delay and average delay. The parameters of the simulation are as follow:

- $\quad$ The buffer of NC and NT are 150Mbit;

- Time interval of burst data obey the exponentially distributed, and the average interval is $1 \mathrm{~ms}$

- $\quad$ Periodic data accounting for $10 \%$ total data;

- The NC application data load is same to NT;

- $\quad$ DBA cycle is $1 \mathrm{~ms}$.

Table 1. the result of the proposed DBA scheme and the traditional scheme

\begin{tabular}{|c|c|c|c|c|c|c|c|c|c|c|}
\hline \multirow[t]{3}{*}{ Node } & \multirow{2}{*}{\multicolumn{2}{|c|}{$\begin{array}{l}\text { Periodic data/us } \\
\text { Delay jitter } \\
\end{array}$}} & \multirow{2}{*}{\multicolumn{2}{|c|}{$\begin{array}{l}\text { Burst data /us } \\
\text { Largest delay }\end{array}$}} & \multicolumn{4}{|c|}{ Applications data/ms (average) } & \multicolumn{2}{|c|}{$\begin{array}{l}\text { throughput } \\
\text { /Gbps(average) }\end{array}$} \\
\hline & & & & & \multicolumn{2}{|c|}{ Largest delay } & \multicolumn{2}{|c|}{ Average delay } & \multirow[t]{2}{*}{ proposed } & \multirow{2}{*}{$\begin{array}{l}\text { Tradition } \\
\text { al }\end{array}$} \\
\hline & proposed & $\begin{array}{l}\text { Tradition } \\
\text { al }\end{array}$ & proposed & $\begin{array}{l}\text { Traditio } \\
\text { nal }\end{array}$ & proposed & $\begin{array}{l}\text { Traditio } \\
\text { nal }\end{array}$ & proposed & $\begin{array}{l}\text { Traditio } \\
\text { nal }\end{array}$ & & \\
\hline 10 & 0 & 0 & & 16.2 & & 313.821 & & 99.2 & 15. & 2.9698 \\
\hline 15 & 0 & 0 & 17.4 & 15.8 & 87.655 & 518.423 & 14.098 & 202.4 & 23.6172 & 2.9852 \\
\hline 20 & 0 & 0 & 18.1 & 15 & 74.227 & 45 & 17.193 & 172.8 & 30.2632 & 2.9252 \\
\hline 25 & 0 & 0 & 18.9 & 15.4 & 90.753 & 574.621 & 23.125 & 220.6 & 38.4052 & 2.8996 \\
\hline 32 & 0 & 0 & 19.8 & 15.2 & 94.991 & 672.231 & 26.289 & 258.8 & 46.1359 & 2.8656 \\
\hline
\end{tabular}

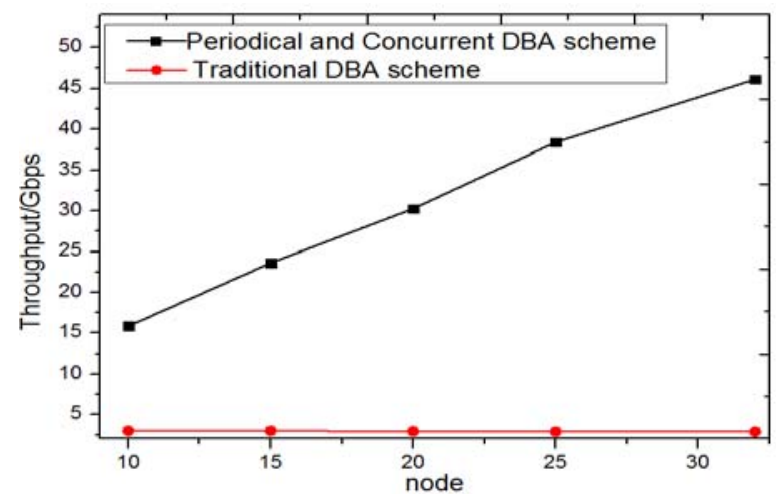

Fig.7 Measured system throughput with different nodes

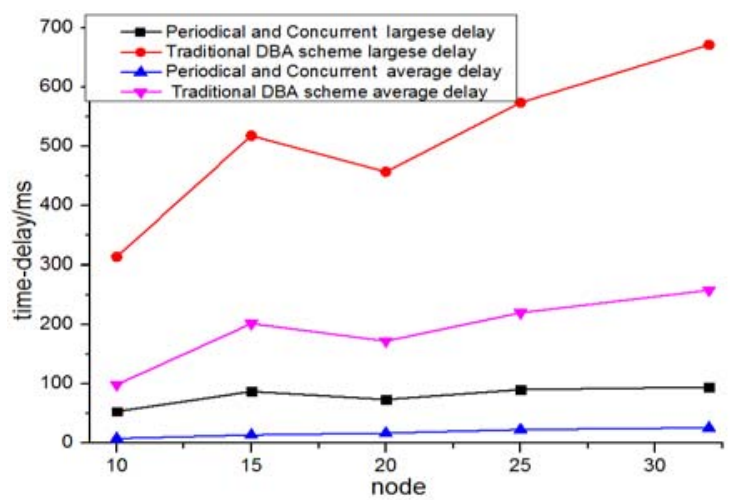

Fig. 8 Measured burst business time-delay with different nodes 
We simulated the throughput, delay jitter of periodic data, the delay of application data, and the largest delay of burst data with different nodes by the periodical and concurrent DBA scheme and tradition DBA scheme, respectively. And the results are shown in Table 1 and Table 2. From the result, the network throughput of 32 nodes can reach $46 \mathrm{Gbps}$ by the proposed DBA scheme while the throughput is $2.8 \mathrm{Gbps}$ by the traditional scheme, as shown in Fig7. And the largest delay and average delay of the prosed DBA scheme is lower than the tradition scheme, as shown in Fig.8.

\section{Conclusions}

Demand for the features of space application network and the requirements of payload data, this paper design a combination of static and dynamic periodical and concurrent dynamic scheduling scheme. The DBA proposal was analyzed theoretically and the performances of switching FC-AE-1553 network based on the periodical and concurrent dynamic scheduling scheme are investigated. Our results show that compared with traditional solutions, the network throughput increased by 10 times, and the time delay could be decreased one order of magnitude. The proposed DBA scheme can provide effective solutions for switching FC-AE-1553 network design and optimization in the future aerospace missions.

\section{References}

[1] PENG Li, Lv cong-min, LIU Ying-chun, HE Yu-feng, "Study of Topology Performance of FC-AE-1553 Network in Space Application", Computer Technology and Development, 2013,Vol.23, No.9, pp.10-13

[2] FANG Liang, ZHAO, Guang-heng, CAO Su-zhi, Evaluation of network transmission performance in FC-AE-1553, Journal of Beijing University of Aeronautics and Astronautics, 2015,41(8):1396-1402

[3] Fiber channel-avionics environment INCTIS TR-31-2002[S].2002.

[4] CAO Su-zhi, ZHANG Shan-cong.Analysis on advanced features of FC-AE-1553(J). Optical Communication Technology, 2010, 34(2): 49-51

[5] FANG Liang, ZHAO, Guang-heng, CAO Su-zhi, Design of heterogeneous FC-AE-1553 network[C], Pro of 2014 CCSSE: $130-134$

[6] FC-AE-1553 INCTIS working draft proposed American national standard for information technology[S].2006

[7] Murdock J R, Koening J R. Opening systems avionics network to replaced MIL-STD-1553[J]. IEEE Aerospace and Electronic Systems Magazine, 2001,Vol.16,No.8 pp.15-19

[8] MaGarry, M.P, Reisslein, M, Investigation of the DBA Algorithm Design Space for EPONs, Journal of Lightwave Technology, 2012, 20(14):2271-2280

[9] DING Fan,XIONG Hua-gang,SONG Li-ru, "Modeling and simulation for FC-AE-1553 network", Computer Engineering and Applications, 2008, Vol.44, No.8, pp.20-24. 